\title{
Characteristics of the chiral phase transition in nonlocal quark models
}

\author{
D. Gómez Dumm ${ }^{1,2}$ and N. N. Scoccola ${ }^{2,3,4}$ \\ ${ }^{1}$ IFLP, Consejo Nacional de Investigaciones Cientificas y Técnicas, Depto. de Física, Universidad Nacional de La Plata, C.C. 67, \\ 1900 La Plata, Argentina \\ ${ }^{2}$ Consejo Nacional de Investigaciones Cientificas y Técnicas, Rivadavia 1917, 1033 Buenos Aires, Argentina \\ ${ }^{3}$ Physics Department, Comisión Nacional de Energía Atómica, Av. Libertador 8250, 1429 Buenos Aires, Argentina \\ ${ }^{4}$ Universidad Favaloro, Solís 453, 1078 Buenos Aires, Argentina
}

(Received 22 October 2004; published 28 July 2005)

\begin{abstract}
The characteristics of the chiral phase transition are analyzed within the framework of chiral quark models with nonlocal interactions in the mean-field approximation. In the chiral limit, we develop a semianalytic framework that allows us to explicitly determine the phase transition curve, the position of the critical points, some relevant critical exponents, etc. For the case of finite current quark masses, we show the behavior of various thermodynamical and chiral response functions across the phase transition.
\end{abstract}

DOI: 10.1103/PhysRevC.72.014909

PACS number(s): $12.39 . \mathrm{Ki}, 11.30 . \mathrm{Rd}, 12.38 . \mathrm{Mh}$

\section{INTRODUCTION}

The behavior of strongly interacting matter under extreme conditions of temperature and/or density has important consequences in nuclear and particle physics as well as in astrophysics and cosmology. From the theoretical point of view, even if a significant progress has been made on the development of $a b$ initio calculations as lattice QCD [1-3], these are not yet able to provide a detailed knowledge of the full QCD phase diagram, and most theoretical approaches rely on the study of low-energy effective models. Qualitatively we expect that chiral symmetry, which is broken at very low temperatures and densities, will be restored as the temperature and density are increased. However, the precise characteristics of this phase transition are still not known. For two massless flavors, most effective approaches to QCD suggest the existence of a tricritical point on the $(T, \mu)$ plane, which separates a first-order phase-transition line found at lower $T$ and larger $\mu$, and a second-order transition line where the chiral restoration occurs for higher $T$ and lower $\mu$. For two light flavors a similar behavior is expected, although the second-order transition line is replaced with a more or less sharp crossover, and, correspondingly, the tricritical point becomes an end point. In any case, for a given effective model that can provide a reasonable successful description of low-energy strong interactions, it is important to obtain as much information as possible about the characteristics of the chiral restoration transition. In previous works $[4,5]$ we have begun the study of chiral restoration in the context of chiral quark models with nonlocal interactions [6], which can be considered as some nonlocal extension of the widely studied Nambu-Jona-Lasinio (NJL) model [7]. In fact, nonlocality arises naturally in the context of several successful approaches to low-energy quark dynamics as, for example, the instanton liquid model [8] and the Schwinger-Dyson resummation techniques [9]. It has been also argued that nonlocal covariant extensions of the NJL model have several advantages over the local scheme, such as, e.g., a natural regularization scheme that automatically preserves the anomalies [10], small next leading order corrections [11], etc. In addition, it has been argued $[12,13]$ that a proper choice of the nonlocal regulator and the model parameters can lead to some form of quark confinement, in the sense that the effective quark propagator has no poles at real energies.

Several studies [13-15] have shown that these nonlocal chiral quark models provide a satisfactory description of the hadron properties at zero temperature and density. The aim of the present work is to complement the analysis of Refs. [4,5], presenting further details about the chiral phase transition within these schemes. Indeed, we show that in the chiral limit it is possible to develop a semianalytic framework that allows us to explicitly determine the phase-transition curve, the position of the critical points, some relevant critical exponents, etc. For the case of finite-current quark masses, we present the behavior of various thermodynamical and chiral response functions across the phase transition. In particular, it is found that thermal and chiral susceptibilities show clear peaks that allow to define a phase-transition temperature as is usually done in lattice calculations.

The paper is organized as follows. In Sec. II we provide a short description of the model and its treatment in the mean-field approximation (MFA). In Sec. III we study the phase transition in the chiral limit by performing the Landau expansion of the free energy. We also obtain the MFA critical exponents. In Sec. IV we present and discuss the behavior of the different thermodynamical and chiral response functions for the case of finite-current quark masses, and we show the corresponding phase diagrams. In Sec. V we present a summary of our main results and conclusions. Finally, some details of the calculations are given in Appendixes A and $\mathrm{B}$.

\section{NONLOCAL CHIRAL QUARK MODELS}

Let us begin by stating the Euclidean action for the nonlocal chiral quark model in the case of two light flavors and SU(2) 
isospin symmetry. We have ${ }^{1}$

$$
S_{E}=\int d^{4} x\left[\bar{\psi}(x)\left(-i \not \partial+m_{c} \mathbb{1}\right) \psi(x)-\frac{G}{2} j_{a}(x) j_{a}(x)\right],
$$

where $\psi=(u d)^{T}$ and $m_{c}$ stands for the $u$ and $d$ current quark mass. The current $j_{a}(x)$ is given by

$$
j_{a}(x)=\int d^{4} y d^{4} z \tilde{r}(y-x) \tilde{r}(x-z) \bar{\psi}(y) \Gamma_{a} \psi(z),
$$

where $\Gamma_{a}=\left(\mathbb{1}, i \gamma_{5} \vec{\tau}\right)$ and the function $\tilde{r}(x-y)$ is a nonlocal regulator. The latter can be translated into momentum space:

$$
\tilde{r}(x-z)=\int \frac{d^{4} p}{(2 \pi)^{4}} e^{-i(x-z) p} r(p) .
$$

In fact, dimensional analysis together with Lorentz invariance implies that $r(p)$ can be a function only of $p^{2} / \Lambda^{2}$, where $\Lambda$ is a cutoff parameter describing the range of the nonlocality in momentum space. Hence we use for the Fourier transform of the regulator the form $r_{\Lambda}\left(p^{2}\right)$ from now on.

From the Euclidean action in Eq. (1), the partition function for the model at zero $T$ and $\mu$ is defined as

$$
\mathcal{Z}_{0}=\int \mathcal{D} \bar{\psi} \mathcal{D} \psi e^{-S_{E}}
$$

We perform now a standard bosonization of the theory, introducing the sigma and pion meson fields $M_{a}(x)=[\sigma(x), \vec{\pi}(x)]$. In this way the partition function can be written as [5]

$$
\mathcal{Z}_{0}=\int \mathcal{D} \sigma \mathcal{D} \pi \operatorname{det} A\left(M_{a}\right) \exp \left[-\frac{1}{2 G} \int \frac{d^{4} p}{(2 \pi)^{4}} M_{a}^{2}(p)\right],
$$

where the operator $A$ reads, in momentum space,

$$
\begin{aligned}
A\left(M_{a}\right)= & \left(-p+m_{c}\right)(2 \pi)^{4} \delta^{(4)}\left(p-p^{\prime}\right) \\
& +r_{\Lambda}\left(p^{2}\right) M_{a}\left(p-p^{\prime}\right) r_{\Lambda}\left(p^{\prime 2}\right) \Gamma_{a} .
\end{aligned}
$$

In what follows we work within the MFA, in which the meson fields are expanded around their translational invariant vacuum expectation values,

$$
\begin{aligned}
\sigma(x) & =\bar{\sigma}+\delta \sigma(x), \\
\pi_{i}(x) & =\delta \pi_{i}(x),
\end{aligned}
$$

and the fluctuations $\delta \sigma(x)$ and $\delta \pi_{i}(x)$ are neglected (vacuum expectation values of the pion fields vanish owing to parity conservation). Within this approximation the determinant in Eq. (5) is formally given by

$$
\begin{aligned}
\operatorname{det} A= & \exp \operatorname{Tr} \log A=\exp V^{(4)} \int \frac{d^{4} p}{(2 \pi)^{4}} \operatorname{tr} \log \\
& \times\left[-\not p+m_{c}+\bar{\sigma} r_{\Lambda}^{2}\left(p^{2}\right)\right],
\end{aligned}
$$

where tr stands for the trace over the Dirac, flavor, and color indices, and $V^{(4)}$ is the four-dimensional volume of the path integral.

\footnotetext{
${ }^{1}$ For simplicity we neglect here possible diquark channels. See Ref. [16] for details on their role in the phase diagram of this type of model.
}

\section{PHASE TRANSITION IN THE CHIRAL LIMIT}

In Refs. [4,5] we have analyzed the chiral restoration within nonlocal chiral quark models for some definite regulators. In particular, we have shown that in the chiral limit this phase transition is a second-order one for low values of the chemical potential $\mu$. In this section we rederive this result, following the so-called classical approach proposed by Landau, in which we expand the free energy in powers of the order parameter (in this case the quark condensate $\langle\bar{q} q\rangle)$ in the vicinity of the critical temperature. This shows the equivalence of the chiral restoration in nonlocal chiral quark models with the corresponding phase transitions taking place in other physical systems, such as ferromagnets or superfluids. As stated, we work within the MFA, which in this context means approximating the path integral in Eq. (5) by its maximum, reached at some saddle point.

In our case, the partition function in the grand canonical ensemble for finite temperature $T$ and chemical potential $\mu$ can be obtained from Eqs. (5) and (9). In these expressions, the integrals over four-momentum space have to be replaced with Matsubara sums according to

$$
\begin{aligned}
& \int \frac{d^{4} p}{(2 \pi)^{4}} F\left(p_{4}, \vec{p}\right) \rightarrow \bigvee_{p} F\left(p_{4}, \vec{p}\right) \\
& \equiv T \sum_{n=-\infty}^{\infty} \int \frac{d^{3} p}{(2 \pi)^{3}} F\left(\omega_{n}-i \mu, \vec{p}\right),
\end{aligned}
$$

where $\omega_{n}$ are the Matsubara frequencies corresponding to fermionic modes, $\omega_{n}=(2 n+1) \pi T$. In the same way volume $V^{(4)}$ is replaced with $V / T$, where $V$ is the three-dimensional volume in coordinate space. As in Refs. [4,5], we are assuming here that the quark interactions depend on only the temperature and chemical potential through the arguments of the regulators. The grand canonical thermodynamical potential per unit volume is thus given by [5]

$$
\begin{aligned}
\omega_{\mathrm{MF}}\left(T, \mu, m_{c}\right) & =-\frac{T}{V} \log \mathcal{Z}_{\mathrm{MF}}\left(T, \mu, m_{c}\right) \\
& =\frac{\bar{\sigma}^{2}}{2 G}-4 N_{c} \bigvee_{p} \log \left[p^{2}+\Sigma^{2}\left(p^{2}\right)\right],
\end{aligned}
$$

where $\Sigma\left(p^{2}\right)=m_{c}+\bar{\sigma} r^{2}\left(p^{2}\right)$ stands for the quark selfenergy, and the mean-field value $\bar{\sigma}\left(T, \mu, m_{c}\right)$ is obtained from the condition

$$
\frac{\partial \omega_{\mathrm{MF}}}{\partial \bar{\sigma}}=0
$$

In fact, $\omega_{\mathrm{MF}}$ turns out to be divergent. The regularization procedure used here amounts to defining

$$
\begin{aligned}
\omega_{\mathrm{MF}}^{(\mathrm{reg})}\left(T, \mu, m_{c}\right)= & \omega_{\mathrm{MF}}\left(T, \mu, m_{c}\right)-\omega_{\text {free }}\left(T, \mu, m_{c}\right) \\
& +\omega_{\text {free }}^{(\text {reg })}\left(T, \mu, m_{c}\right)+\omega_{0},
\end{aligned}
$$

where $\omega_{\text {free }}^{(\mathrm{reg})}\left(T, \mu, m_{c}\right)$ is the regularized expression for the thermodynamical potential of a free fermion gas, and $\omega_{0}$ is a constant fixed by the condition $\omega_{\mathrm{MF}}^{(\mathrm{reg})}=0$ at $T=\mu=0$ (see Appendix A for details). 
In the analogy with a ferromagnetic system, the chiral condensate can be identified with the magnetization per unit volume, $\langle\bar{q} q\rangle \longleftrightarrow-M / V$, whereas the current quark mass $m_{c}$ plays the role of the external magnetic field $H$. We have

$$
\langle\bar{q} q\rangle=\frac{1}{2}\left(\frac{\partial \omega}{\partial m_{c}}\right)_{T, \mu},
$$

where the $\frac{1}{2}$ factor results from the fact that this relation holds for each quark flavor separately, i.e., $q=u, d$, although they have a common mass $m_{c}$. In the chiral limit, the existence of a second-order phase transition for a fixed value of $\mu$ implies that the condensate $\langle\bar{q} q\rangle$ goes to zero when the temperature $T$ approaches from below a given critical value $T_{c}(\mu)$, above which we have $\langle\bar{q} q\rangle=0$ and the chiral symmetry is restored. Thus, for $T \sim T_{c}(\mu)$ and to leading order in $m_{c}$, we can perform the Landau expansion (see Appendix A)

$$
\begin{aligned}
\omega_{\mathrm{MF}}^{(\mathrm{reg})}\left(T, \mu, m_{c}\right)= & \omega_{0}+\omega_{\text {free }}^{(\mathrm{reg})}\left(T, \mu, m_{c}=0\right) \\
& +A(T, \mu)\langle\bar{q} q\rangle^{2}+C(T, \mu)\langle\bar{q} q\rangle^{4} \\
& +2 m_{c}\langle\bar{q} q\rangle+\mathcal{O}\left(\langle\bar{q} q\rangle^{6},\langle\bar{q} q\rangle^{3} m_{c}, m_{c}^{2}\right),
\end{aligned}
$$

where the coefficients $A$ and $C$ are given by

$$
\begin{aligned}
A(T, \mu)= & \frac{1}{4 N_{c}^{2} S_{11}^{2}(T, \mu)}\left[\frac{1}{8 G}-N_{c} S_{21}(T, \mu)\right], \\
C(T, \mu)= & \frac{S_{42}(T, \mu)}{128 N_{c}^{3} S_{11}^{4}(T, \mu)}-\frac{S_{32}(T, \mu)}{32 N_{c}^{4} S_{11}^{5}(T, \mu)} \\
& \times\left[\frac{1}{8 G}-N_{c} S_{21}(T, \mu)\right],
\end{aligned}
$$

with

$$
S_{m n}(T, \mu)=\oiint_{p} \frac{r_{\Lambda}^{2 m}\left(p^{2}\right)}{p^{2 n}} .
$$

The regularized thermodynamical potential for a massless fermion gas — second term on the right-hand side of Eq. (15) can be calculated by evaluation of the integral in Eq. (A2) in the massless case. We obtain

$$
\omega_{\text {free }}^{(\mathrm{reg})}(T, \mu, 0)=-\frac{N_{c}}{3}\left[\frac{7 \pi^{2}}{30} T^{4}+T^{2} \mu^{2}+\frac{1}{2 \pi^{2}} \mu^{4}\right] .
$$

In the limit $m_{c}=0$, it can be seen [17] that for $C>0$ the system undergoes a second-order phase transition at a critical temperature $T_{c}(\mu)$, obeying $A\left[T_{c}(\mu), \mu\right]=0$. This implies

$$
S_{21}\left[T_{c}(\mu), \mu\right]=\frac{1}{8 G N_{c}},
$$

which defines a second-order transition curve in the $(T, \mu)$ plane. As is described in Appendix B, for sufficiently small (but relevant) values of $T$ and $\mu$ the Matsubara sum implicit in $S_{21}\left[T_{c}(\mu), \mu\right]$ can be analytically worked out. This leads to a simple relation between $T_{c}$ and $\mu$, namely

$$
\frac{\pi^{2}}{3}\left[\frac{T_{c}(\mu)}{\Lambda}\right]^{2}+\left(\frac{\mu}{\Lambda}\right)^{2}=\beta_{0}-\frac{\pi^{2}}{N_{c}} \frac{1}{G \Lambda^{2}},
$$

where $\beta_{0}=\Lambda^{-2} \int d p p r_{\Lambda}^{4}\left(p^{2}\right)$ is a dimensionless quantity that depends on only the shape of the regulator. Note that this relation generalizes that obtained in Ref. [18] for the NJL model. We also point out that, for a given value of $\mu$ and temperatures that are close to the critical value $T_{c}(\mu)$ obtained from Eq. (19), we have

$$
A(T, \mu)=\lambda t,
$$

where

$$
t \equiv\left[T-T_{c}(\mu)\right] / T_{c}(\mu), \quad \lambda=\frac{T_{c}^{2}(\mu)}{48 N_{c} S_{11}^{2}\left[T_{c}(\mu), \mu\right]} .
$$

We note in passing that, once the Landau expansion has been established, it is a usual textbook exercise [17] to derive the critical exponents ruling the behavior of the specific heat, the order parameter $\langle\bar{q} q\rangle$, and the chiral susceptibility near the second-order critical points:

$$
\begin{array}{rlrl}
\left.c_{V, \mu, m_{c}}\right|_{m_{c}=0} & \sim|t|^{-\alpha},\left.\quad\langle\bar{q} q\rangle\right|_{m_{c}=0} & \sim|t|^{\beta}, \\
\left.\chi_{V, T, \mu}\right|_{m_{c}=0} & \sim|t|^{-\gamma},\left.\quad\langle\bar{q} q\rangle\right|_{t=0} \sim m_{c}^{\delta} .
\end{array}
$$

Here the specific heat and the chiral susceptibility are defined as

$$
\begin{aligned}
c_{V, \mu, m_{c}} & =-T\left[\frac{\partial^{2} \omega\left(T, \mu, m_{c}\right)}{\partial T^{2}}\right]_{\mu, m_{c}} \\
& =T\left[\frac{\partial s\left(T, \mu, m_{c}\right)}{\partial T}\right]_{\mu, m_{c}}, \\
\chi_{V, T, \mu} & =-\frac{1}{2}\left[\frac{\partial^{2} \omega\left(T, \mu, m_{c}\right)}{\partial m_{c}^{2}}\right]_{T, \mu} \\
& =-\left[\frac{\partial\langle\bar{q} q\rangle\left(T, \mu, m_{c}\right)}{\partial m_{c}}\right]_{T, \mu},
\end{aligned}
$$

where $s\left(T, \mu, m_{c}\right)$ is the entropy density. As expected, we obtain the mean-field critical exponents, ${ }^{2}$

$$
\alpha=0, \quad \beta=\frac{1}{2}, \quad \gamma=1, \quad \delta=3,
$$

a result that was under discussion in the somewhat related Dyson-Schwinger models of QCD [19]. Equation (26) imply that the chiral susceptibility diverges at $T=T_{c}$. In the case of specific heat, there is no divergence but a finite discontinuity at $m_{c}=0, t=0$,

$$
\left.c_{V, \mu, m_{c}}\right|_{t=0^{-}}=\left.c_{V, \mu, m_{c}}\right|_{t=0^{+}}+\frac{N_{c} T_{c}^{3}}{36 S_{42}},
$$

where $\left.c_{V, \mu, m_{c}}\right|_{t=0^{+}}$is the specific heat at constant $\mu$ that is due to the free fermion gas pressure $p_{\text {free }}=-\omega_{\text {free }}^{\text {(reg) }}(T, \mu, 0)$, evaluated at $T=T_{c}$. From Eq. (18) we get

$$
\left.c_{V, \mu, m_{c}}\right|_{t>0}=\frac{2}{3} N_{c} T\left(\mu^{2}+\frac{7 \pi^{2}}{5} T^{2}\right) .
$$

As stated, the transition remains second order as long as $C\left[T_{c}(\mu), \mu\right]>0$. This is expected to be the case for low values of the chemical potential. However, if $\mu$ is increased, we could reach a point at which the coefficient $C$ vanishes. Beyond this point, called "tricritical," the system undergoes a first-order

\footnotetext{
${ }^{2}$ At the tricritical point, the mean field critical exponents are $\alpha=$ $\frac{1}{2}, \beta=\frac{1}{4}, \gamma=1$, and $\delta=5$.
} 


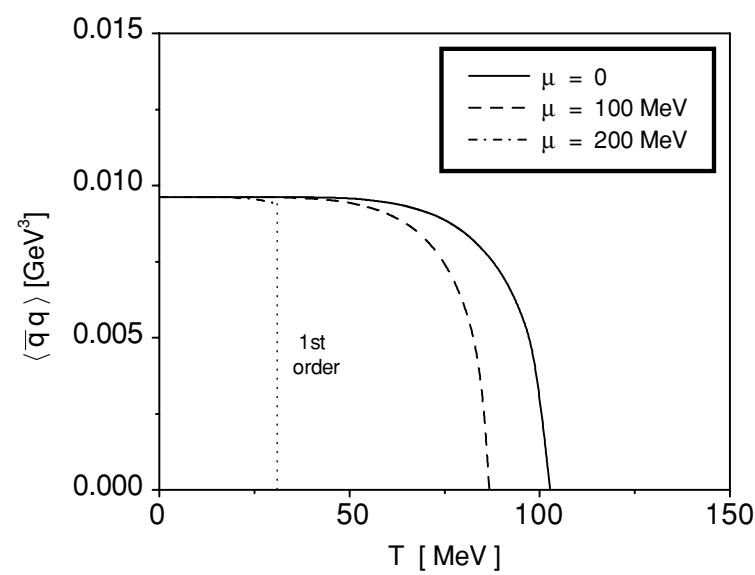

(a)

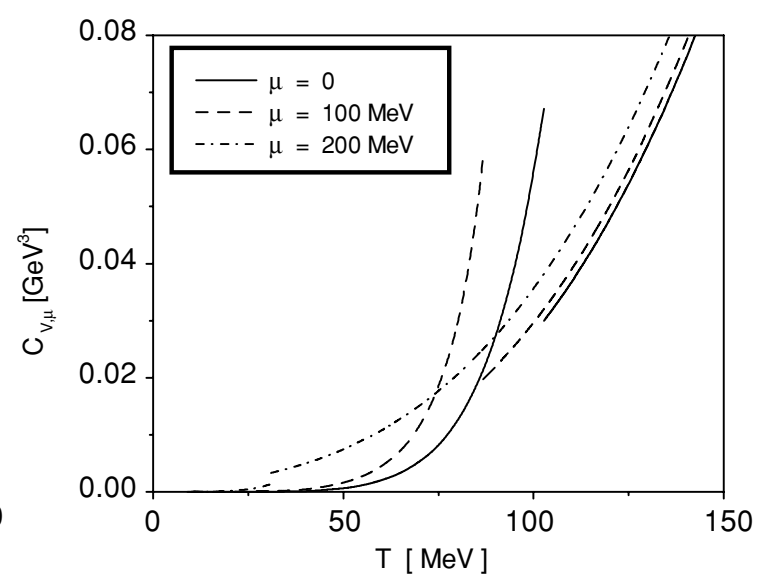

(b)

FIG. 1. (a) Chiral condensate vs. temperature and (b) specific heat $c_{V, \mu}$ vs temperature. Solid, dashed, and dashed-dotted curves correspond to $\mu=0, \mu=100$, and $200 \mathrm{MeV}$, respectively.

phase transition (the order parameter $\langle\bar{q} q\rangle$ is discontinuous at the corresponding critical temperature). In this way, the tricritical point is obtained from the conditions $A(T, \mu)=$ $C(T, \mu)=0$ or, equivalently,

$$
S_{42}\left[T_{c}(\mu), \mu\right]=0 .
$$

Again, for sufficiently small (but relevant) values of $T$ and $\mu$ the Matsubara sum in $S_{42}$ can be analytically worked out (see Appendix B), leading to

$$
\begin{aligned}
S_{42}(T, \mu)= & \frac{1}{8 \pi^{2}}\left\{\beta_{1}(T / \Lambda)^{2}\left[\frac{\pi^{2}}{3}+(\mu / T)^{2}\right]\right. \\
& \left.+\beta_{2}-f(\mu / T)-\log (T / \Lambda)\right\},
\end{aligned}
$$

where

$$
\begin{aligned}
& \beta_{1}=-\left.8 \Lambda^{2} \frac{d r_{\Lambda}\left(p^{2}\right)}{d p^{2}}\right|_{p=0}, \\
& \beta_{2}=\int_{0}^{\infty} \frac{d p}{p}\left[r_{\Lambda}^{8}\left(p^{2}\right)-e^{-4 p^{2}}\right]+\frac{1}{2}(\gamma-1)-\log 2 \pi,
\end{aligned}
$$

and $f(x)$, which satisfies $f(0)=0$, is given by Eq. (B10) of Appendix B. It is interesting to note the similarity between our expressions in Eqs. (20), (29), and (30) and those obtained in Ref. [20] within a very different theoretical approach. A brief discussion on the subject is also included in Appendix B.

Let us consider for definiteness a nonlocal model in which the regulator is a Gaussian function,

$$
r_{\Lambda}\left(p^{2}\right)=\exp \left(-p^{2} / 2 \Lambda^{2}\right)
$$

In this case we have $\beta_{1}=4$, and the integral in $\beta_{2}$ vanishes. In addition, it is easily seen that $S_{42}(T, 0)$ is a positive function of $T / \Lambda$, which implies that in the chiral limit the model leads to a second-order chiral phase transition for vanishing chemical potential. The transition remains second order when $\mu$ is increased up to the tricritical point, and the corresponding transition line in the $(T, \mu)$ plane can be immediately obtained from Eq. (20), with $\beta_{0}=1 / 4$. The critical temperature at $\mu=0$ is thus given by

$$
T_{c}(0)=\frac{\sqrt{3}}{2 \pi} \Lambda\left(1-\frac{4 \pi^{2}}{N_{c} G \Lambda^{2}}\right)^{1 / 2} .
$$

We illustrate these features by considering a particular parameter set, namely $\Lambda=760 \mathrm{MeV}$ and $G=30 \mathrm{GeV}^{-2}$. These are the parameters corresponding to Set II in Ref. [5], leading to $T_{c}(0)=102 \mathrm{MeV}$. The second-order phase transition is clearly shown in Fig. 1(a), where the solid curve represents the chiral condensate for $\mu=0$ as a function of $T$. Now, by using Eq. (29), we can find the position of the tricritical point, which in this case is found to be located at $(T, \mu)=$ (72 MeV, $133 \mathrm{MeV}$ ). In Fig. 1(a), dashed and dashed-dotted curves show the behavior of the chiral condensate for $\mu=$ 100 and $200 \mathrm{MeV}$, below and above the tricritical point, respectively. Both the values for $T_{c}(0)$ and the position of the tricritical point are found to be in very good agreement with those numerically obtained in Ref. [5]. Finally, the described effect on the specific heat is shown in Fig. 1(b), where we plot $c_{V, \mu}$ as a function of the temperature for $\mu=0,100$, and $200 \mathrm{MeV}$.

The result in Eq. (33) is useful for pointing out a generic feature of this type of model in the chiral limit. In fact, it is not difficult to show [13] that, in such a limit, for zero $T$ and $\mu$ one obtain expressions for the pion decay constant $f_{\pi}^{(0)}$ and the chiral condensate $\langle\bar{q} q\rangle^{(0)}$ such that $f_{\pi}^{(0)} / \Lambda$ and $\langle\bar{q} q\rangle^{(0)} / \Lambda$ depend on only the combination $G \Lambda^{2}$. To get a ratio $f_{\pi}^{(0)} /\langle\bar{q} q\rangle^{(0)} \simeq 0.4$, as required by phenomenology, these expressions lead to $G \Lambda^{2} \simeq 16$. If this value is now replaced in Eq. (33) we get $T_{c}(0) / \Lambda \simeq 0.12$, which is somewhat large, but still lies within the expected range of validity. However, if we impose the critical temperature $T_{c}(0)$ to be approximately equal to $170 \mathrm{MeV}$, as suggested by lattice QCD calculations, we get $\Lambda \simeq 1.4 \mathrm{GeV}$, which, together with $G \Lambda^{2} \simeq 16$, enhances $f_{\pi}^{(0)}$ and $\langle\bar{q} q\rangle^{(0)}$ up to roughly $40 \%$ above 
the corresponding phenomenological values. Thus it can be generically said that, if in the chiral limit-and within the MFA - we want to satisfy the phenomenological constraints on the values of $f_{\pi}$ and $\langle\bar{q} q\rangle$, the nonlocal models under consideration lead to a relatively low critical temperature $T_{c}(0)$. Of course, this might be modified by finite quark mass effects and/or beyond MFA corrections.

\section{PHASE TRANSITION AND RESPONSE FUNCTIONS FOR FINITE QUARK MASS}

In this section we concentrate on the analysis of the phase transition for finite-current quark masses in the isospin limit. For this purpose we consider different thermodynamical response functions. In particular, it will be seen that, for reasonable values of the current quark mass $m_{c}$, the chiral susceptibilities show clear peaks that can be used to define the transition curves in the crossover region.

The response functions are basically given by the second derivatives of the Helmholtz free energy $F(T, V, \bar{N})$. The latter is related to the thermodynamical potential by

$$
F(V, T, \bar{N})=[\Omega(V, T, \mu)+2 \bar{N} \mu]_{\mu=\mu(V, T, \bar{N})},
$$
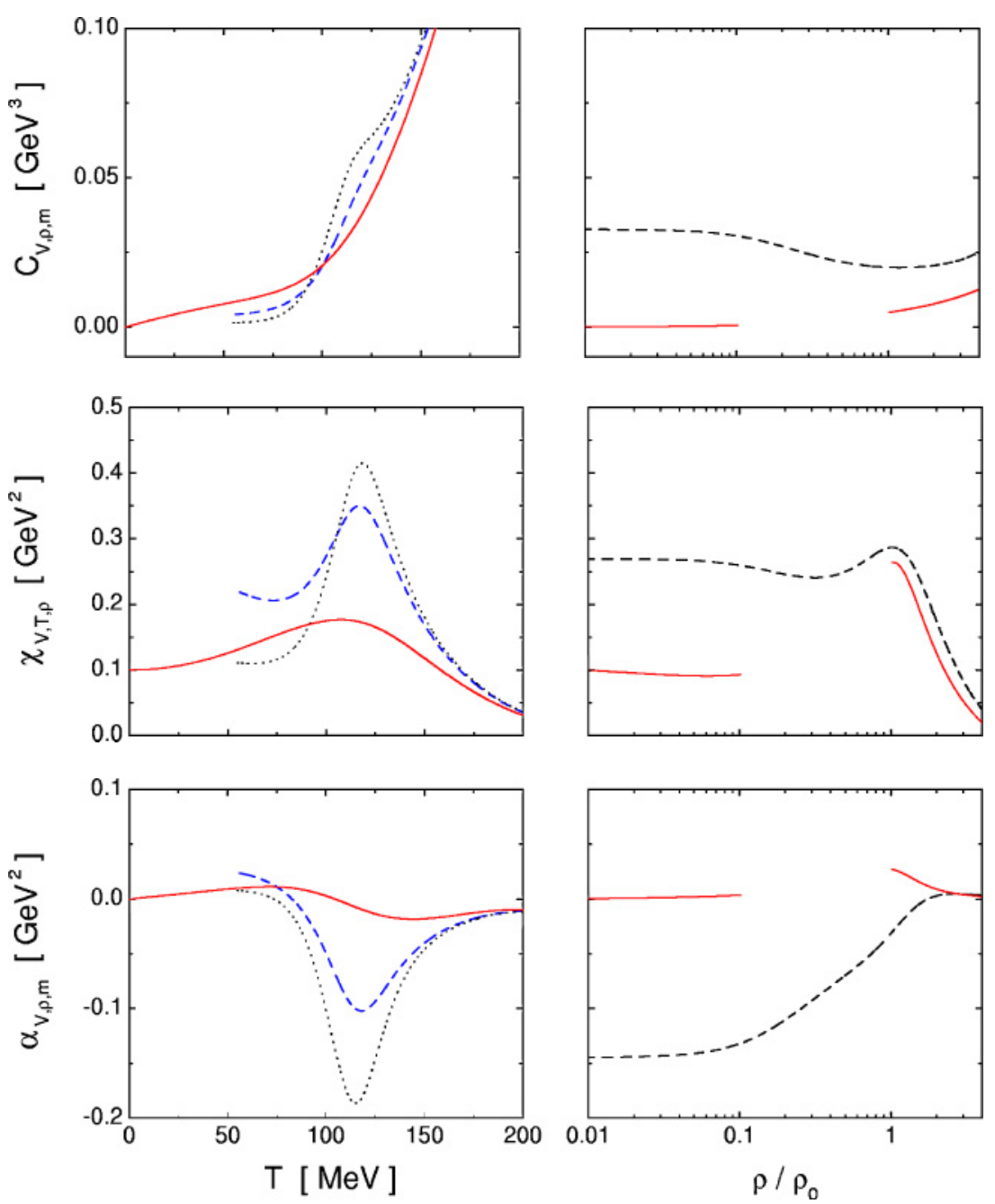

where $\bar{N}=-\frac{1}{2}(\partial \Omega / \partial \mu)_{V, T}$ (as in the case of the quark condensate, the $\frac{1}{2}$ factor comes from defining $\bar{N}=\bar{N}_{u}=\bar{N}_{d}$ ). We consider, as before, the limit of a large system in which $\Omega(V, T, \mu)=V \omega(T, \mu), F(V, T, \bar{N})=V f(T, \bar{N} / V)$; thus, instead of average particle number $\bar{N}$, our quantities will be given in terms of the average particle density $\rho=\bar{N} / V$. We can define three independent thermodynamical response functions, namely the specific heat at fixed volume and particle number $c_{V, \rho, m}$, the isothermal compressibility $\kappa_{T, \rho, m_{c}}$, and the coefficient of thermal expansion $\alpha_{p, \rho, m_{c}}$. These are given by

$$
\begin{aligned}
c_{V, \rho, m_{c}} & =-\frac{T}{V}\left[\frac{\partial^{2} F\left(T, V, \bar{N}, m_{c}\right)}{\partial T^{2}}\right]_{V, \bar{N}, m_{c}} \\
& =T\left[\frac{\partial s\left(T, \rho, m_{c}\right)}{\partial T}\right]_{\rho, m_{c}}, \\
\frac{1}{\kappa_{T, \rho, m_{c}}} & =V\left[\frac{\partial^{2} F\left(T, V, \bar{N}, m_{c}\right)}{\partial V^{2}}\right]_{T, \bar{N}, m_{c}} \\
& =\rho\left[\frac{\partial p\left(T, \rho, m_{c}\right)}{\partial \rho}\right]_{T, m_{c}},
\end{aligned}
$$

FIG. 2. (Color online) Some response functions for fixed $\rho$, corresponding to parameter Set II. In the left-hand panels curves are given as functions of $T$, with $\rho / \rho_{0}$ fixed at three representative values, namely 0.25 (dotted), 0.75 (dashed), and 2.0 (solid). Right-hand panels show the curves as functions of $\rho / \rho_{0}$ for $T$ fixed at $50 \mathrm{MeV}$ (solid) and $100 \mathrm{MeV}$ (dashed). 

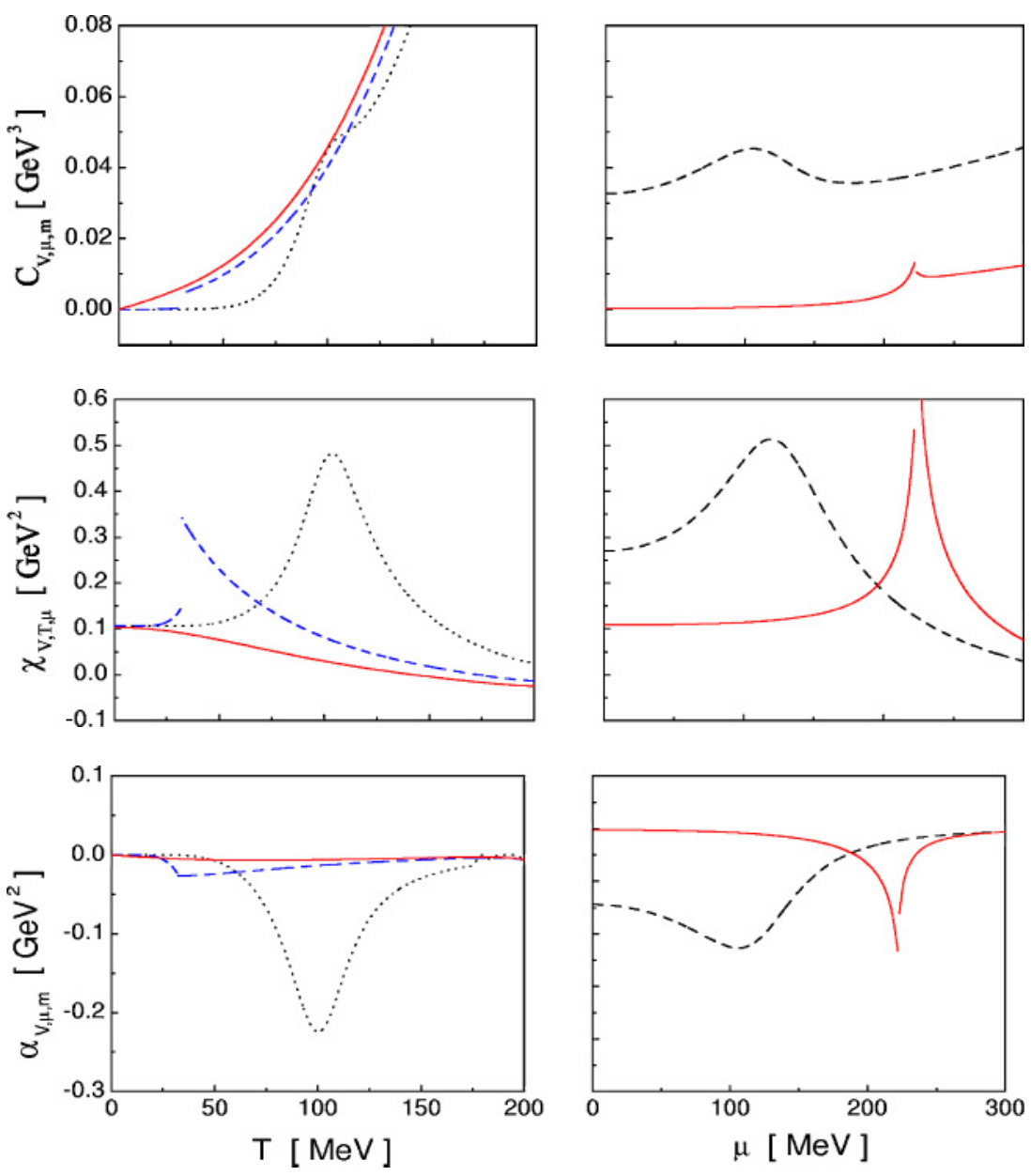

FIG. 3. (Color online) Some response functions for fixed $\mu$, corresponding to parameter Set II. In the left-hand panels curves are given as functions of $T$, with $\mu$ fixed at three representative values, namely $100 \mathrm{MeV}$ (dotted), $250 \mathrm{MeV}$ (dashed), and $300 \mathrm{MeV}$ (solid). Righthand panels show the curves as functions of $\mu$ for $T$ fixed at $50 \mathrm{MeV}$ (solid) and $100 \mathrm{MeV}$ (dashed).

$$
\begin{aligned}
\alpha_{p, \rho, m_{c}} & =\frac{1}{V}\left(\frac{\partial V}{\partial T}\right)_{p, \bar{N}, m_{c}} \\
& =-\kappa_{T, \rho, m_{c}}\left[\frac{\partial^{2} F\left(T, V, \bar{N}, m_{c}\right)}{\partial V \partial T}\right]_{\bar{N}, m_{c}} \\
& =\kappa_{T, \rho, m_{c}}\left[\frac{\partial p\left(T, \rho, m_{c}\right)}{\partial T}\right]_{\rho, m_{c}} .
\end{aligned}
$$

There are also other thermodynamical response functions that can be defined, such as the specific heat at constant pressure $c_{p, \rho, m_{c}}$ and the adiabatic compressibility $\kappa_{s, \rho, m_{c}}$. However, these can be written in terms of the three quantities in Eqs. (35)-(37).

Note that we have explicitly included the dependence of the free energy on the current quark mass $m_{c}$. In fact, we can still define some extra response functions associated with the chiral transition by differentiating the free energy with respect to $m_{c}$. In the analogy with magnetic systems, these would be the specific heats at constant magnetization and constant applied magnetic field, $C_{M}, C_{H}$, the isothermal and adiabatic susceptibilities $\chi_{T}, \chi_{S}$, and the coefficient of thermal magnetization, $\alpha_{H}$. As before, these quantities are related to each other, leaving only two new independent response functions. Here we choose to consider those analogous to the isothermal susceptibility and the coefficient of thermal magnetization; thus we define

$$
\begin{aligned}
\chi_{V, T, \rho} & =-\frac{1}{2 V}\left[\frac{\partial^{2} F\left(T, V, \bar{N}, m_{c}\right)}{\partial m_{c}^{2}}\right]_{T, V, \bar{N}} \\
& =-\left[\frac{\partial\langle\bar{q} q\rangle\left(T, \rho, m_{c}\right)}{\partial m_{c}}\right]_{T, \rho}, \\
\alpha_{V, \rho, m_{c}} & =-\frac{1}{2 V}\left[\frac{\partial^{2} F\left(T, V, \bar{N}, m_{c}\right)}{\partial m_{c} \partial T}\right]_{V, \bar{N}} \\
& =-\left[\frac{\partial\langle\bar{q} q\rangle\left(T, \rho, m_{c}\right)}{\partial T}\right]_{\rho, m_{c}} .
\end{aligned}
$$

Let us show the numerical results for these quantities for the case of a nonlocal Gaussian regulator. We have chosen the parameter set $\Lambda=760 \mathrm{MeV}, G=30 \mathrm{GeV}^{-2}$, and $m_{c}=7.7 \mathrm{MeV}$, which corresponds to Set II in the notation of Ref. [5]. It can be seen that these parameters lead to the empirical values of the pion mass and decay constant and provide reasonable results for the chiral quark condensate and the quark self-energy $\Sigma(0)$ at zero $T$ and $\mu$. In Fig. 2 we show the curves corresponding to the specific heat $c_{V, \rho, m_{c}}$ and the chiral response functions $\chi_{V, T, \rho}$ and $\alpha_{V, \rho, m_{c}}$. In the left-hand panels, these functions are plotted versus the temperature for three representative values of the density (the quoted values refer to nuclear matter density, $\rho_{0} \simeq 1.3 \times 10^{6} \mathrm{MeV}^{3}$ ). Note 

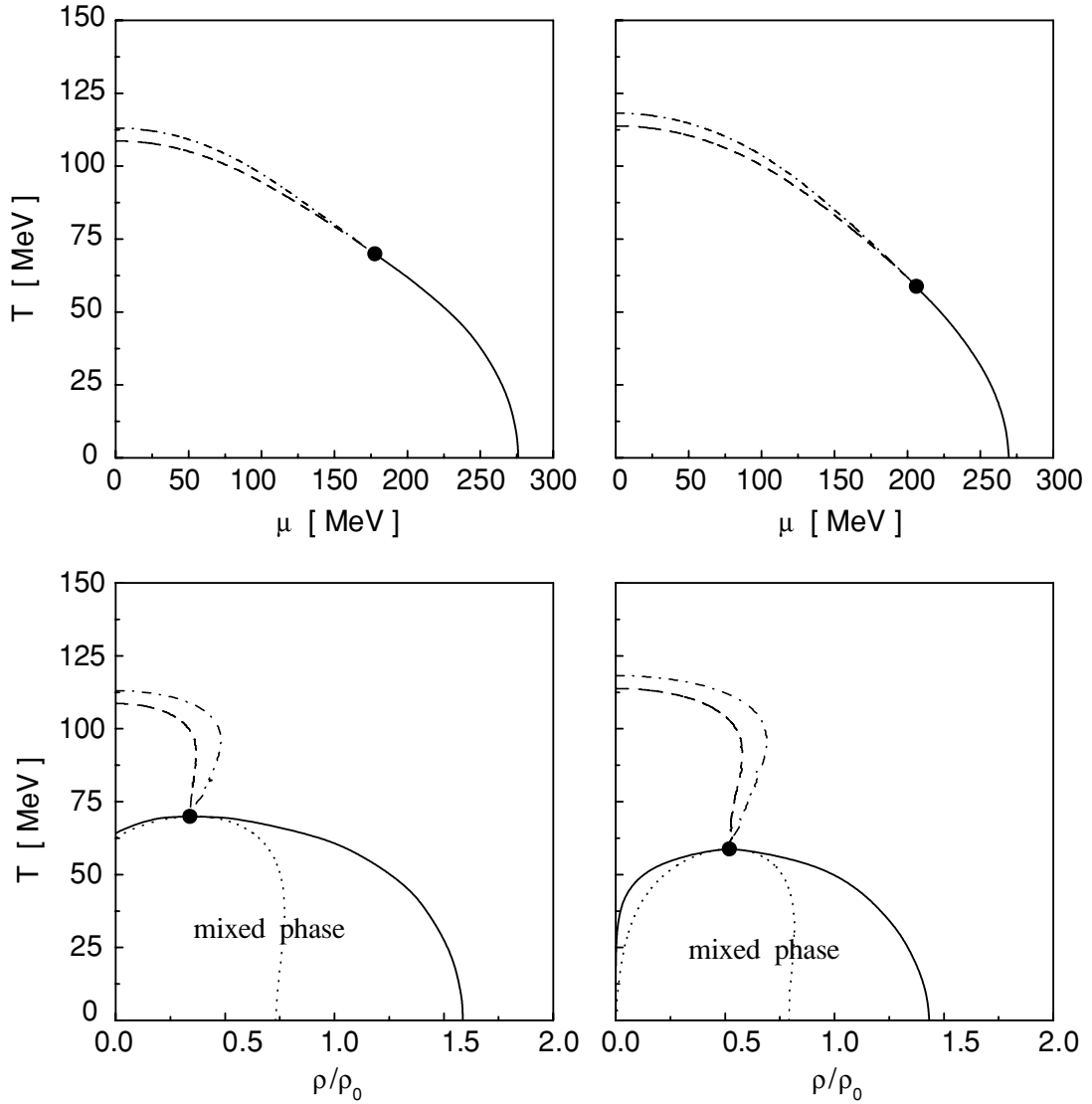

FIG. 4. Phase diagram showing the chiral transition curves in the $T-\mu$ plane (upper panels) and the $T-\rho$ plane (lower panels), for parameter Sets I (left) and II (right). Solid curves correspond to first-order phase-transition curves, and dashed (dashed-dotted) curves show the crossover curves obtained from the peaks in the chiral (thermal) susceptibilities. The dotted curves in the lower panels represent the spinodals, and the fat dots indicate in each case the position of the end point. that for low nonzero densities the system is homogeneous only for temperatures that exceed a critical value. Below this limit, as we will see, there is a region where phases with broken and restored chiral symmetry coexist. In the right-hand panels, the same response functions are plotted as functions of the density, fixing the temperature at 50 and $100 \mathrm{MeV}$. Once again, for low temperatures there is a mixed-phase region, and as a consequence the functions are not well defined at intermediate densities. We have also analyzed the response functions corresponding to the parameter set given by $G=50 \mathrm{GeV}^{-2}, m_{c}=10.5 \mathrm{MeV}$, and $\Lambda=627 \mathrm{MeV}$, or Set I in the notation of Ref. [5]. Sets I and II might be interpreted as confining and nonconfining, respectively, in which "confinement" is understood in the sense that the pole structure of the quark propagator does not allow quarks to materialize on-shell in Minkowski space [12,13]. The curves in the case of Set I do not differ qualitatively from those shown in Fig. 2; therefore they have not been included here.

Now let us pay special attention to the responses of the chiral condensate (order parameter of the phase transition) at fixed temperature and chiral quark mass (or "magnetization"). These are given by Eqs. (38) and (39), and their behavior as functions of the temperature is shown in the second and third rows of Fig. 2 (left-hand panels). As is well known, susceptibilities are particularly useful for analyzing the phasetransition features. In the present model, as shown in previous works [4,5], for low temperatures the system undergoes a first-order chiral phase transition, which turns into a smooth crossover for temperatures exceeding a given "end point." In this crossover region, the transition is characterized by the presence of respective peaks in the mentioned susceptibilities, the height and sharpness of these peaks giving a measure of the crossover steepness. The same can be observed if we look at the chiral and thermal susceptibilities for fixed $\mu$, which are given by the second derivatives of the thermodynamical potential and are in fact the natural quantities to deal with in the grand canonical ensemble. The definition of the chiral susceptibility $\chi_{V, T, \mu}$ has already been given in Eq. (25), and the thermal susceptibility at constant $\mu$ is defined as

$$
\begin{aligned}
\alpha_{V, \mu, m_{c}} & =-\frac{1}{2}\left[\frac{\partial^{2} \omega\left(T, \mu, m_{c}\right)}{\partial m_{c} \partial T}\right]_{\mu} \\
& =-\left[\frac{\partial\langle\bar{q} q\rangle\left(T, \mu, m_{c}\right)}{\partial T}\right]_{\mu, m_{c}} .
\end{aligned}
$$

The curves showing the behavior of the susceptibilities $\chi_{V, T, \mu}$ and $\alpha_{V, \mu, m}$ as functions of the temperature and the chemical potential are shown in Fig. 3 (the chosen parameter set is the same as that in Fig. 2). For completeness we also include the curves for the specific heat at constant $\mu$, previously introduced in Sec. III (where the case of varying $m_{c}$ was discussed). In a natural way, the peaks in the curves for $\chi_{V, T, \mu}$ and $\alpha_{V, \mu, m_{c}}$ can be used to define the position at which the chiral transition occurs. Thus we can extend the phase-space diagram to include the crossover transition curves in addition to the first-order ones. This is represented in Fig. 4, 

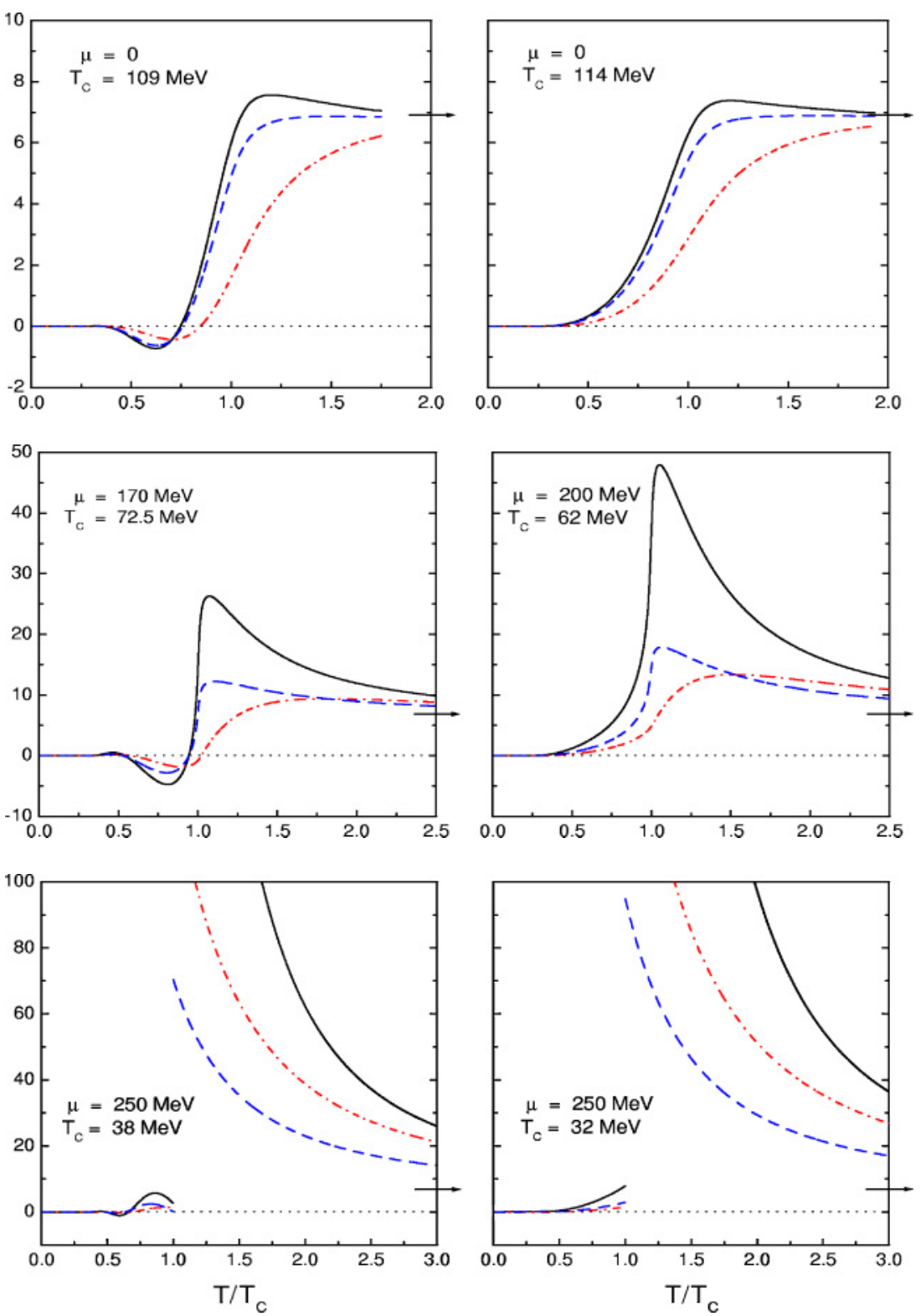

FIG. 5. (Color online) Numerical results for the scaled quantities $\varepsilon / T^{4}$ (solid curves), $\frac{3}{4} s / T^{3}$ (dashed curves), and $3 p / T^{4}$ (dashed-dotted curves). Curves are given as functions of $T / T_{c}$ for fixed values of $\mu$ that are indicated in each case together with the respective critical temperatures. The arrows show the asymptotic values, which correspond to a free fermion system. Leftand right-hand panels show the results obtained for Sets I and II, respectively. where crossover curves obtained from the peaks in $\chi_{V, T, \mu}$ and $\alpha_{V, \mu, m_{c}}$ have been represented by dashed and dotted curves, respectively. Although chiral and thermal susceptibilities lead to slightly different points, it is seen that the transition region is well defined. Solid curves correspond to the first-order phase transition, and the fat dots indicate the end points. Left- and right-hand panels show the results for parameter Sets I and II, respectively, and upper (lower) panels show the phase-space transition curves in the $T-\mu(T-\rho)$ plane. Note that in the $T-\rho$ phase diagrams there is a region below the first-order transition lines where both phases are allowed. The latter can be interpreted [21] as a zone in which droplets containing light quarks of mass $m_{c}$ coexist with a gas of constituent, massive quarks. The dotted curves inside this zone are the spinodals, i.e., the boundaries of the region in which the energetically unfavored solutions can exist as metastable states.

Our results for the phase-transition curves in the $T-\mu$ plane are qualitatively similar to those obtained from lattice
QCD calculations [2,3], although both the critical temperature at $\mu=0$ and the end-point temperature turn out to be relatively low in our case (see discussion at the end of Sec. III). For the sake of comparison, it is also interesting to analyze the curvature of the phase boundary at $\mu=0$. This is an appropriate quantity to be studied in lattice QCD, where the main problem in the analysis of the $T-\mu$ phase diagram is the inclusion of a finite real chemical potential. Recent lattice calculations [1] yielded $\left.T_{c}\left(d^{2} T_{c} / d \mu^{2}\right)\right|_{\mu=0}=-0.14 \pm 0.06$, whereas the result obtained within the standard NJL model (up to some finite-current quark mass corrections) gives a value of about -0.40 [22]. In our model, the curvature can be calculated numerically from the results plotted in Fig. 4, leading to values of about -0.26 and -0.30 for Sets I and II, respectively. In the chiral limit the corresponding calculation can be carried out from the analytical expression in Eq. (20), leading to $\left.T_{c}\left(d^{2} T_{c} / d \mu^{2}\right)\right|_{\mu=0}=-3 / \pi^{2} \simeq-0.304$. 
Finally, let us quote the numerical results for the behavior of the energy density, the entropy density, and the pressure as functions of temperature. The corresponding curves are shown in Fig. 5, where we plot the scaled quantities $\varepsilon / T^{4}, \frac{3}{4} s / T^{3}$, and $3 p / T^{4}$ versus the relative temperature $T / T_{c}$ for Sets I and II and different values of the chemical potential. The arrows indicate the value corresponding to a free fermion system in the large $T$ limit, given by $7 \pi^{2} / 10$ - see Eq. (18). Note that for Set $I$ there is a range of temperatures below $T_{c}$ for which all three quantities are negative. This might be taken as a further indication that for this parameter set there is a sort of "confinement": Somewhat below $T_{c}$ hadronic degrees of freedom ought to be included, and the system cannot be simply treated as a quark gas. This represents a qualitative difference between Sets I and II. It is interesting to see that, in spite of this fact, the behavior at the transition region is relatively similar in both cases.

\section{SUMMARY AND OUTLOOK}

In this paper we have presented further details of the chiral phase transition within chiral quark models including nonlocal interactions in the MFA. In the chiral limit, our analysis allows us to obtain semianalytical expressions for the transition curve and the location of the critical points. For the case of finite-current quark masses, we have studied the behavior of various thermodynamical and chiral response functions across the phase transition. In the crossover region the thermal and chiral susceptibilities display clear peaks that allow us to define a transition temperature in the same way as is usually done in lattice calculations.

The resulting phase diagrams are qualitatively similar to those obtained within other frameworks, such as, e.g., the standard NJL model, the Bag model, and lattice QCD. In the nonlocal schemes studied here, however, the transition temperature at $\mu=0$ is found to be somewhat lower than the expected value $T_{c}(0) \simeq 170 \mathrm{MeV}$ once the model parameters are fitted so as to reproduce both the empirical values of $m_{\pi}$ and $f_{\pi}$ and the phenomenological value of the light quark condensates at zero temperature and density. In fact, this result may be improved if alternative ways of including nonlocality are included, such as, e.g., regulator schemes inspired in one-gluon exchange processes [23], or through the inclusion of beyond MFA contributions. Work in this direction is currently in progress.

\section{ACKNOWLEDGMENTS}

We thank M. Malheiro for useful discussions. This work has been partially supported by Consejo Nacional de Investigaciones Cientificas y Técnicas and ANPCyT, under grants PIP 02368, PICT00-03-08580, and PICT02-03-10718.

\section{APPENDIX A: EXPANSION OF THE THERMODYNAMICAL POTENTIAL IN POWERS OF $\langle\bar{q} q\rangle$}

The grand canonical thermodynamical potential defined by Eq. (11) turns out to be divergent. Here we have regularized it by subtracting the expression corresponding to a system of free fermions of mass $m_{c}$ and adding it in a regularized form. We have thus

$$
\begin{aligned}
\omega_{\mathrm{MF}}^{(\mathrm{reg})}\left(T, \mu, m_{c}\right)= & \frac{\bar{\sigma}^{2}}{2 G}-4 N_{c} \bigvee_{p} \log \left[\frac{p^{2}+\Sigma^{2}\left(p^{2}\right)}{p^{2}+m_{c}^{2}}\right] \\
& +\omega_{\text {free }}^{(\mathrm{reg})}\left(T, \mu, m_{c}\right)+\omega_{0}
\end{aligned}
$$

where

$$
\begin{aligned}
\omega_{\text {free }}^{(\mathrm{reg})}\left(T, \mu, m_{c}\right)= & -4 N_{c} T \int \frac{d^{3} p}{(2 \pi)^{3}}\left\{\log \left[1+e^{-(E-\mu) / T}\right]\right. \\
& \left.+\log \left[1+e^{-(E+\mu) / T}\right]\right\}
\end{aligned}
$$

where $E=\sqrt{p^{2}+m_{c}^{2}}$ and $\omega_{0}$ is a constant fixed by the condition that the thermodynamical potential vanish at zero $T$ and $\mu$. We point out that this regularization prescription relies on the well-behaved shape of the regulator in $\Sigma\left(p^{2}\right)$. Alternative schemes can be applied in other cases; see, e.g. [24].

Let us assume that in the chiral limit, $m_{c}=0$, the order parameter $\langle\bar{q} q\rangle$ vanishes at a given temperature $T=T_{c}$. We are interested in the description of the situation in the vicinity of this temperature; hence it is useful to carry out a double expansion of $\omega_{\mathrm{MF}}^{(\mathrm{reg})}$ in powers of $\langle\bar{q} q\rangle$ and $m_{c}$. For $m_{c}=0$, we can perform the expansion of $\omega_{\mathrm{MF}}^{(\mathrm{reg})}$ in powers of $\langle\bar{q} q\rangle$ by taking into account the derivatives

$$
\begin{aligned}
& \left.\frac{\partial^{2} \omega_{\mathrm{MF}}}{\partial\langle\bar{q} q\rangle^{2}}\right|_{\langle\bar{q} q\rangle=0}=\left.\frac{\partial^{2} \omega_{\mathrm{MF}}}{\partial \sigma^{2}}\left(\frac{\partial\langle\bar{q} q\rangle}{\partial \sigma}\right)^{-2}\right|_{\langle\bar{q} q\rangle=0}, \\
& \left.\frac{\partial^{4} \omega_{\mathrm{MF}}}{\partial\langle\bar{q} q\rangle^{4}}\right|_{\langle\bar{q} q\rangle=0}=\frac{\partial^{4} \omega_{\mathrm{MF}}}{\partial \sigma^{4}}\left(\frac{\partial\langle\bar{q} q\rangle}{\partial \sigma}\right)^{-4}-\left.4 \frac{\partial^{2} \omega_{\mathrm{MF}}}{\partial \sigma^{2}}\left(\frac{\partial\langle\bar{q} q\rangle}{\partial \sigma}\right)^{-5} \frac{\partial^{3}\langle\bar{q} q\rangle}{\partial \sigma^{3}}\right|_{\langle\bar{q} q\rangle=0}, \\
& \left.\frac{\partial \omega_{\mathrm{MF}}}{\partial\langle\bar{q} q\rangle}\right|_{\langle\bar{q} q\rangle=0}=\left.\frac{\partial^{3} \omega_{\mathrm{MF}}}{\partial\langle\bar{q} q\rangle^{3}}\right|_{\langle\bar{q} q\rangle=0}=\left.\frac{\partial^{5} \omega_{\mathrm{MF}}}{\partial\langle\bar{q} q\rangle^{5}}\right|_{\langle\bar{q} q\rangle=0}=0 .
\end{aligned}
$$


Because $\langle\bar{q} q\rangle=0$ implies $\sigma=0$, it is easy to see that the preceeding partial derivatives are given by

$$
\begin{aligned}
& \left.\frac{\partial\langle\bar{q} q\rangle}{\partial \sigma}\right|_{\sigma=0}=-4 N_{c} S_{11}(T, \mu), \\
& \left.\frac{\partial^{3}\langle\bar{q} q\rangle}{\partial \sigma^{3}}\right|_{\sigma=0}=24 N_{c} S_{32}(T, \mu), \\
& \left.\frac{\partial^{2} \omega_{\mathrm{MF}}}{\partial \sigma^{2}}\right|_{\sigma=0}=\frac{1}{G}-8 N_{c} S_{21}(T, \mu), \\
& \left.\frac{\partial^{4} \omega_{\mathrm{MF}}}{\partial \sigma^{4}}\right|_{\sigma=0}=48 N_{c} S_{42}(T, \mu),
\end{aligned}
$$

where the functions $S_{m n}(T, \mu)$ are defined by

$$
S_{m n}(T, \mu)=\bigcup_{p} \frac{r^{2 m}\left(p^{2}\right)}{p^{2 n}} .
$$

On the other hand, for $m_{c} \neq 0$, we have

$$
\left.\frac{\partial \omega_{\mathrm{MF}}}{\partial m_{c}}\right|_{m_{c}=0}=2\langle\bar{q} q\rangle .
$$

Taking into account this relation, together with Eqs. (A4) and (A3), we easily arrive at the result shown in Eq. (15).

\section{APPENDIX B: EVALUATION OF MATSUBARA SUMS}

In this appendix we show how to work out the Matsubara sums $S_{m 1}$ and $S_{m 2}$, which are defined by Eq. (A5). These sums appear in the Landau expansion of the thermodynamical potential near the critical temperature; see Eq. (15).

To carry out the calculations, we take into account the analysis in Ref. [5], where Cauchy's theorem was used to convert the Matsubara sums into an integral plus a sum over pole residues. In the case of a function $F\left(p^{2}\right)$ that has only simple poles and no cuts in the complex plane, we obtain [5]

$$
\begin{aligned}
y_{p} F\left(p^{2}\right)= & \int \frac{d^{4} p}{(2 \pi)^{4}} F\left(p^{2}\right) \\
& +2 \int \frac{d^{3} p}{(2 \pi)^{3}} \operatorname{Re} \sum_{\substack{R_{k}>-\mu \\
I_{k} \geqslant 0}} \gamma_{k} \operatorname{Res}\left[\mathcal{F}(z) ; z_{k}\right] \\
& \times\left[n_{+}\left(z_{k}+\mu\right)+n_{-}\left(z_{k}+\mu\right)\right]
\end{aligned}
$$

where the function $\mathcal{F}$ is defined as $\mathcal{F}(z) \equiv F\left[(-i z-i \mu)^{2}+\right.$ $\left.|\vec{p}|^{2}\right], z_{k}=R_{k}+i I_{k}$ are the residues of this function in the complex plane $z$, and the coefficient $\gamma_{k}$ is defined as $\gamma_{k}=1 / 2$ for $I_{k}=0$ and $\gamma_{k}=1$ otherwise. We have also introduced here (complex) occupation number functions $n_{ \pm}(z)$, defined by

$$
n_{ \pm}(z)=\frac{1}{1+\exp [(z \mp \mu) / T]} .
$$

In the case of the sum $S_{21}(T, \mu)$, the corresponding function $\mathcal{F}_{21}(z)$ is given by

$$
\mathcal{F}_{21}(z)=\frac{r_{\Lambda}^{4}\left[-(z+\mu)^{2}+|\vec{p}|^{2}\right]}{-(z+\mu)^{2}+|\vec{p}|^{2}},
$$

which has only two simple poles located at $z^{ \pm}=-\mu \pm|\vec{p}|$, with residues $\mp(2|\vec{p}|)^{-1}$. Therefore Eq. (B1) can in principle be applied. There is, however, a subtle point to be taken into account. The derivation of Eq. (B1) assumes that $|\mathcal{F}(z)| \rightarrow 0$ when $\operatorname{Re} z \rightarrow \infty$, which is in fact true for all the situations considered in Ref. [5]. However, depending on the specific form of the regulator, this condition might not be satisfied by the function $\mathcal{F}_{21}(z)$. If this is the case, Eq. (B1) can still be applied, provided that $T, \mu \ll \Lambda$ (see subsequent discussion).

Assuming that Eq. (B1) holds, we get

$$
\begin{aligned}
S_{21}(T, \mu)= & y_{p} \frac{r_{\Lambda}^{4}\left(p^{2}\right)}{p^{2}}=\frac{1}{8 \pi^{2}} \int_{0}^{\infty} d p p r_{\Lambda}^{4}\left(p^{2}\right) \\
& -\frac{1}{4 \pi^{2}} \int_{0}^{\infty} d p p\left[n_{+}(p)+n_{-}(p)\right] .
\end{aligned}
$$

The last integral in this expression can be worked out analytically. We have

$$
\begin{aligned}
& \int_{0}^{\infty} d p p\left[n_{+}(p)+n_{-}(p)\right] \\
& \quad=-T^{2}\left[\operatorname{Li}_{2}\left(-e^{\mu / T}\right)+\operatorname{Li}_{2}\left(-e^{-\mu / T}\right)\right]=\frac{\pi^{2} T^{2}}{6}+\frac{\mu^{2}}{2}
\end{aligned}
$$

which, together with Eq. (B4), leads to the result quoted in Eq. (20). As we have mentioned, these relations are valid only for sufficiently low values of $T, \mu$ compared with the cutoff scale $\Lambda$. In the case of the Gaussian regulator we have checked that, for $T \leqslant \Lambda / 6$ and $\mu \leqslant \Lambda / 4$, Eq. (20) is verified with an accuracy of less that $1 \%$. In the case of the Lorentzian regulator the region of validity is somewhat smaller, but at the same time the relevant cutoff parameter is larger than in the Gaussian case [5]. As a conclusion, we find that in all cases considered here Eq. (20) can be taken to be valid with very good approximation for the values of $T$ and $\mu$ of physical interest.

A similar analysis can be carried out for the sum $S_{42}(T, \mu)$. Here the situation is somewhat more complicated, as we have to deal with double poles and Eq. (B1) is no longer valid. We have instead

$$
\begin{aligned}
S_{42}(T, \mu)= & \bigcup_{p} \frac{r^{8}\left(p^{2}\right)}{p^{4}} \\
= & \int \frac{d^{4} p}{(2 \pi)^{4}} F\left(p^{2}\right)+\int \frac{d^{3} p}{(2 \pi)^{3}}\left\{\operatorname{Res}\left[\frac{\mathcal{F}(z)}{1+e^{z / T}}\right]_{z^{+}}\right. \\
& \left.-\operatorname{Res}\left[\frac{\mathcal{F}(z)}{1+e^{-z / T}}\right]_{z^{-}}\right\},
\end{aligned}
$$

where $F\left(p^{2}\right)=r_{\Lambda}^{8}\left(p^{2}\right) / p^{4}, \quad \mathcal{F}(z)=F\left[-\left(z+\mu^{2}\right)+|\vec{p}|^{2}\right]$. Evaluating the residues leads to

$$
\begin{aligned}
S_{42}(T, \mu)= & \frac{1}{8 \pi^{2}} \int_{0}^{\infty} d p\left\{\frac{1}{2 \pi}\left[\int_{-\infty}^{\infty} d q \frac{r_{\Lambda}^{8}\left(p^{2}+q^{2}\right)}{\left(p^{2}+q^{2}\right)^{2}}\right]\right. \\
& -\frac{1}{p}\left[n_{+}(p)+n_{-}(p)\right]
\end{aligned}
$$




$$
\begin{aligned}
& -4 m r_{\Lambda}^{\prime}(0) p\left[n_{+}(p)+n_{-}(p)\right] \\
& \left.-\frac{1}{T}\left[n_{+}(p)+n_{-}(p)-n_{+}^{2}(p)-n_{-}^{2}(p)\right]\right\},
\end{aligned}
$$

where $r_{\Lambda}^{\prime}(0)=d r_{\Lambda}\left(p^{2}\right) /\left.d p^{2}\right|_{p^{2}=0}$. The first two terms lead to divergent integrals that we can work out with the help of a definite regulator, e.g., the Gaussian one, by writing

$$
r_{\Lambda}^{8}\left(x^{2}\right)=r_{\Lambda}^{8}\left(x^{2}\right)-e^{-4 x^{2} / \Lambda^{2}}+e^{-4 x^{2} / \Lambda^{2}} .
$$

For the Gaussian regulator, the sum of the divergent integrals in Eq. (B7), properly regularized, is given by

$$
\begin{gathered}
\int_{0}^{\infty} d p\left\{\frac{1}{2 \pi}\left[\int_{-\infty}^{\infty} d q \frac{e^{-4\left(p^{2}+q^{2}\right) / \Lambda^{2}}}{\left(p^{2}+q^{2}\right)^{2}}\right]-\frac{1}{p}\left[n_{+}(p)+n_{-}(p)\right]\right\} \\
=\frac{1}{2}+\frac{\gamma}{2}-\log (\pi \sqrt{m})-\log (T / \Lambda)-f(\mu / T),
\end{gathered}
$$

where

$$
f(x)=2 \sinh ^{2}(x / 2) \int_{0}^{\infty} \frac{d y}{y} \frac{\tanh (y / 2)}{\cosh x+\cosh y} .
$$

Finally, the integral of the last term in Eq. (B7) can be explicitly performed, giving simply

$$
\int_{0}^{\infty} d p\left[n_{+}(p)+n_{-}(p)-n_{+}^{2}(p)-n_{-}^{2}(p)\right]=T .
$$

The previous result in Eq. (B5), together with Eqs. (B7)-(B11), leads to the expression quoted in Eq. (30). Again this relation is strictly valid for $T, \mu \ll \Lambda$. For the Gaussian regulator we have checked that it still holds up to $1 \%$ in the region $T \leqslant \Lambda / 9, \mu \leqslant \Lambda / 5$. As before, this is also the case for the Lorentzian regulator for the values of $T$ and $\mu$ relevant for our analysis.

It is interesting to point out the similarity between our equations determining the second-order transition line and the tricritical point, and those obtained in Ref. [20]. In that article, the authors address the study of the $T-\mu$ phase diagram by using a different approach, in which they propose a large flavor number expansion and a resummed renormalization scheme. It can be seen that the results in Ref. [20], Eqs. (13) and (14), have the same form as our expressions if we take the limit $\lambda=0$, which in the context of Ref. [20] means neglecting the contributions from the meson sector (this should be analogous to the MFA considered here). Moreover, the validity of these results is also limited to relatively low values of $T$ and $\mu$ in view of the presence of a Landau pole arising from the renormalization procedure.
[1] C. R. Allton, S. Ejiri, S. J. Hands, O. Kaczmarek, F. Karsch, E. Laermann, and C. Schmidt, Phys. Rev. D 68, 014507 (2003).

[2] Z. Fodor and S. D. Katz, J. High Energy Phys. 03 (2002) 014; 04 (2004) 050.

[3] F. Karsch and E. Laermann, in Quark-Gluon Plasma III, edited by R. C. Hwa and X. N. Wang (World Scientific, Singapore, 2004).

[4] I. General, D. Gómez Dumm, and N. N. Scoccola, Phys. Lett. B506, 267 (2001).

[5] D. Gómez Dumm and N. N. Scoccola, Phys. Rev. D 65, 074021 (2002).

[6] G. Ripka, Quarks Bound by Chiral Fields (Oxford University Press, Oxford, UK, 1997).

[7] U. Vogl and W. Weise, Prog. Part. Nucl. Phys. 27, 195 (1991); S. Klevansky, Rev. Mod. Phys. 64, 649 (1992); T. Hatsuda and T. Kunihiro, Phys. Rep. 247, 221 (1994).

[8] T. Schafer and E. V. Schuryak, Rev. Mod. Phys. 70, 323 (1998).

[9] C. D. Roberts and A. G. Williams, Prog. Part. Nucl. Phys. 33, 477 (1994); C. D. Roberts and S. M. Schmidt, ibid. 45, S1 (2000).

[10] E. Ruiz Arriola and L. L. Salcedo, Phys. Lett. B450, 225 (1999).

[11] G. Ripka, Nucl. Phys. A683, 463 (2001); R. S. Plant and M. C. Birse, ibid. A703, 717 (2002).

[12] M. Stingl, Phys. Rev. D 34, 3863 (1986) [Erratum-ibid. 36, 651 (1987)]; H. J. Munczek, Phys. Lett. B175, 215 (1986); C. J. Burden, C. D. Roberts, and A. G. Williams, ibid. B285, 347 (1992); G. Krein, C. D. Roberts, and A. G. Williams,
Int. J. Mod. Phys. A 7, 5607 (1992); D. Blaschke, G. Burau, Y. L. Kalinovsky, P. Maris, and P. C. Tandy, ibid. 16, 2267 (2001).

[13] R. D. Bowler and M. C. Birse, Nucl. Phys. A582, 655 (1995); R. S. Plant and M. C. Birse, ibid. A628, 607 (1998).

[14] A. Scarpettini, D. Gómez Dumm, and N. N. Scoccola, Phys. Rev. D 69, 114018 (2004).

[15] W. Broniowski, B. Golli, and G. Ripka, Nucl. Phys. A703, 667 (2002); A. H. Rezaeian, N. R. Walet, and M. C. Birse, Phys. Rev. C 70, 065203 (2004).

[16] R. S. Duhau, A. G. Grunfeld, and N. N. Scoccola, Phys. Rev. D 70, 074026 (2004).

[17] See, e.g., K. Huang, Statistical Mechanics, (Wiley, New York, 1987), Sec. 17.4.

[18] T. M. Schwarz, S. P. Klevansky, and G. Papp, Phys. Rev. C 60, 055205 (1999).

[19] D. Blaschke, A. Holl, C. D. Roberts, and S. Schmidt, Phys. Rev. C 58, 1758 (1998); A. Holl, P. Maris, and C. D. Roberts, ibid. 59, 1751 (1999).

[20] A. Jakovác, A. Patkós, Zs. Szép, and P. Szépfalusy, Phys. Lett. B582, 179 (2004).

[21] J. Berges and K. Rajagopal, Nucl. Phys. B538, 215 (1999).

[22] M. Buballa, Phys. Rep. 407, 205 (2005).

[23] S. M. Schmidt, D. Blaschke, and Y. L. Kalinovsky, Phys. Rev. C 50, 435 (1994).

[24] D. Blaschke, C. D. Roberts, and S. M. Schmidt, Phys. Lett. B425, 232 (1998). 